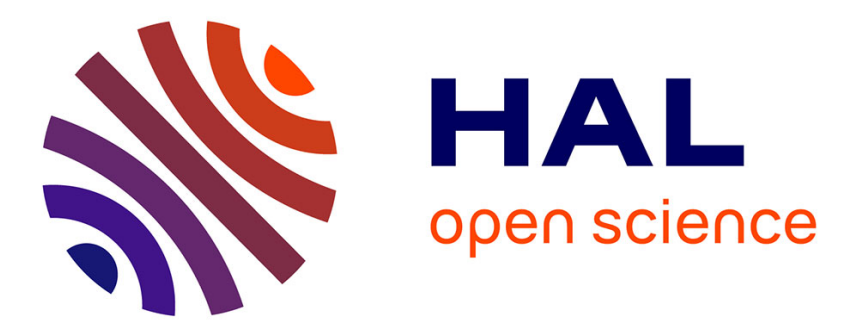

\title{
Oscillations ultrarapides de l'absorption et de la biréfringence photoinduites observées par une technique de modulation temporelle
}

\author{
J. Chesnoy, A. Mokhtari
}

\section{- To cite this version:}

J. Chesnoy, A. Mokhtari. Oscillations ultrarapides de l'absorption et de la biréfringence photoinduites observées par une technique de modulation temporelle. Revue de Physique Appliquée, 1987, 22 (12), pp.1743-1747. 10.1051/rphysap:0198700220120174300 . jpa-00245734

HAL Id: jpa-00245734

https://hal.science/jpa-00245734

Submitted on 1 Jan 1987

HAL is a multi-disciplinary open access archive for the deposit and dissemination of scientific research documents, whether they are published or not. The documents may come from teaching and research institutions in France or abroad, or from public or private research centers.
L'archive ouverte pluridisciplinaire HAL, est destinée au dépôt et à la diffusion de documents scientifiques de niveau recherche, publiés ou non, émanant des établissements d'enseignement et de recherche français ou étrangers, des laboratoires publics ou privés. 


\title{
Oscillations ultrarapides de l'absorption et de la biréfringence photoinduites observées par une technique de modulation temporelle
}

\author{
J. Chesnoy et A. Mokhtari \\ Laboratoire d'Optique Quantique du C.N.R.S., Ecole Polytechnique, 91128 Palaiseau Cedex, France
}

(Reçu le $1^{\text {er }}$ juin 1987, accepté le 27 juin 1987)

\begin{abstract}
Résumé. - Une mesure de la relaxation du dichroïsme et de la biréfringence photoinduits dans un colorant a été effectuée par « excitation-sondage » en utilisant un laser femtoseconde à haute cadence. La mise au point d'une technique de modulation du délai temporel avec détection synchrone du signal à la fréquence harmonique nous a permis d'observer avec une grande précision un comportement oscillatoire amorti. Cette technique est utile pour améliorer le rapport signal sur bruit de ce faible signal superposé à un grand fond lentement variable et nous a permis d'observer pour la première fois une modulation de plusieurs térahertz de l'effet Kerr optique.
\end{abstract}

\begin{abstract}
The relaxation of photoinduced dichroism and birefringence was studied in a dye by a « pumpprobe " measurement using a high repetition rate femtosecond dye laser. A new technique permitted the precise observation of oscillatory damped behaviour, by modulation of the time delay and synchronous detection of the signal at the harmonic frequency. This technique is useful to improve the signal to noise ratio of this weak signal superimposed on a large slowly varying background and has permitted the first observation of modulation at several terahertz in an optical Kerr effect measurement.
\end{abstract}

\section{Introduction.}

Les lasers délivrant des impulsions brèves permettent, en utilisant des techniques d'excitation-sondage, d'étudier la dynamique des systèmes matériels, avec une résolution temporelle limitée par la durée des impulsions lumineuses [1]. En utilisant des lasers femtosecondes, des mesures de saturation d'absorption et de biréfringence induite résolues dans le temps permettent d'observer les mouvements ultrapides des molécules en phase dense [2-4]. Ainsi, Rosker, Wise et Tang [5] ont observé récemment une modulation à plusieurs térahertz de la saturation d'absorption dans des molécules de colorants. Une difficulté de ce type de mesures vient de la faible amplitude de ces modulations rapides superposées à un important signal plus lentement variable. En utilisant un laser à haute cadence, une variation d'absorption induite inférieure à $10^{-5}$ peut être détectée, mais à cause du bruit du laser à basse fréquence, une précision meilleure que $10^{-2}$ sur un signal d'absorption induite peut difficilement être dépassée. Une analyse de ce type de comportement expérimental nécessite pourtant une grande précision de mesure.
Nous présentons ici une technique permettant d'avoir accès préférentiellement au comportement à haute fréquence d'une réponse mesurée dans une expérience d'excitation-sondage : le délai temporel entre les deux impulsions lumineuses d'excitation et de sondage est modulé sinusoïdalement tandis que le signal est mesuré à la fréquence harmonique par détection synchrone. Cette technique, permettant d'améliorer de façon importante le rapport signal sur bruit d'une expérience de saturation d'absorption, nous a d'autre part permis d'observer, pour la première fois, des modulations à plusieurs térahertz de l'effet Kerr optique dans des molécules de colorant en solution, décrivant en temps réel la vibration moléculaire.

\section{Montage expérimental.}

La figure 1 décrit le montage expérimental permettant d'observer le dichroïsme ou la biréfringence induits dans un jet de colorant (le vert de malachite). Les impulsions du laser femtoseconde (durant 5060 fs FWHM émises à une cadence de $50 \mathrm{MHz}$ ) [6] sont focalisées sur l'échantillon absorbant sur un diamètre d'environ $15 \mu \mathrm{m}$, avec une polarisation à 


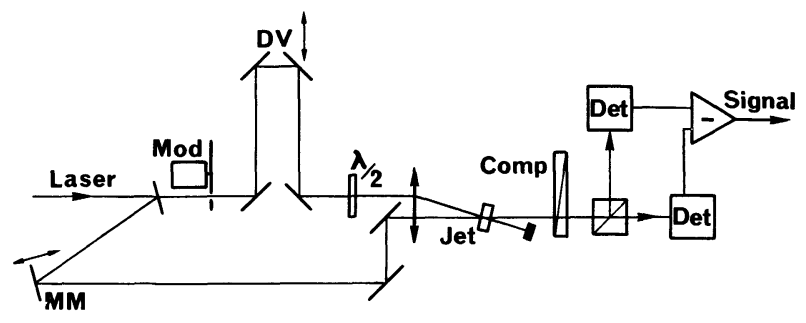

Fig. 1. - L'impulsion femtoseconde incidente est divisée en deux. L'impulsion de pompe, intense, est focalisée sur un échantillon de colorant (le vert de malachite) situé dans un jet, après rotation de sa polarisation de $45^{\circ}$ par une lame $\lambda / 2$. Le faisceau de sonde est lui aussi focalisé dans l'échantillon. Sa polarisation est analysée par une technique linéaire et différentielle à l'aide de deux détecteurs (Det). Un compensateur de Babinet Soleil (comp) permet d'analyser soit la rotation de polarisation (dichroïsme) soit l'ellipticité (biréfringence) induite en fonction du délai temporel (DV) variable entre excitation et sondage. Le miroir mobile MM est utilisé dans la technique de modulation du délai temporel.

[The incident femtosecond pulse is divided in two. The intense pump pulse is focussed into a dye sample (malachite green) situated in a jet after rotation of its polarisation by $45^{\circ}$ with a $\lambda / 2$ plate. The probe beam is focussed into the same part of the sample. Its polarisation is analysed by a differential technique with two detectors (det). A Babinet-Soleil compensator (comp) allows the linear detection of the polarisation rotation (dichroism) or of the induced ellipticity (birefringence) as a function of the variable time delay (DV) between pump and probe. The moving miror MM is used in the time delay modulation technique.]

$45^{\circ}$ de l'horizontale. Un faisceau sonde, peu intense, est focalisé après un délai variable avec une polarisation horizontale sur l'échantillon. La polarisation de ce faisceau sonde est analysée à l'aide d'un compensateur de Babinet et d'un polarisateur. Deux détecteurs de mesure sont équilibrés pour délivrer un signal différentiel. Le compensateur, ajusté à une différence de marche de $\lambda / 2$ et un angle de $22^{\circ} 5$, donne accès à la différence des coefficients d'absorption $\left(\alpha_{\|}-\alpha_{\perp}\right)$ entre directions parallèle et perpendiculaire à la polarisation d'excitation. Quand la différence de marche est ajustée à $\lambda / 4$ et l'angle à $45^{\circ}$, la différence des signaux délivrés par les deux détecteurs donne accès à la biréfringence induite [3] (effet Kerr optique) $\left(n_{\|}-n_{\perp}\right)$, différence entre les indices de réfraction parallèlement et perpendiculairement à la polarisation d'excitation. Ces mesures peuvent être effectuées en fonction du délai variable $\tau$, en modulant l'intensité du faisceau pompe et en utilisant une détection synchrone ou bien directement en sommant sur un grand nombre de passages. Le signal $S(\tau)$ alors obtenu est relié à la fonction réponse $R(t)$ du système $\left(\alpha_{\|}(t)-\alpha_{\perp}(t)\right)$ pour le dichroïsme ou $\left(n_{\|}(t)-n_{\perp}(t)\right)$ pour la biréfringence induite) par :

$$
S(\tau) \propto \int_{-\infty}^{\infty} R\left(t^{\prime}\right) C\left(\tau-t^{\prime}\right) \mathrm{d} t^{\prime}
$$

en négligeant l'«artefact cohérent » [1]. La résolution temporelle est déterminée par la fonction de corrélation $C(t)$, fonction d'appareil de l'expérience :

$$
C(t)=\int_{-\infty}^{\infty} I_{\mathrm{p}}\left(t^{\prime}\right) I_{\mathrm{s}}\left(t^{\prime}-t\right) \mathrm{d} t^{\prime}
$$

calculée avec les profils d'intensité des impulsions laser $I_{\mathrm{p}}$ de pompe et $I_{\mathrm{s}}$ de sonde (identiques dans notre cas) et qui est mesurée expérimentalement par somme de fréquences dans un cristal non linéaire.

La figure 2 donne les résultats des mesures effectuées sur le colorant vert de malachite en solution dans l'eau avec en référence, au-dessus, la fonction

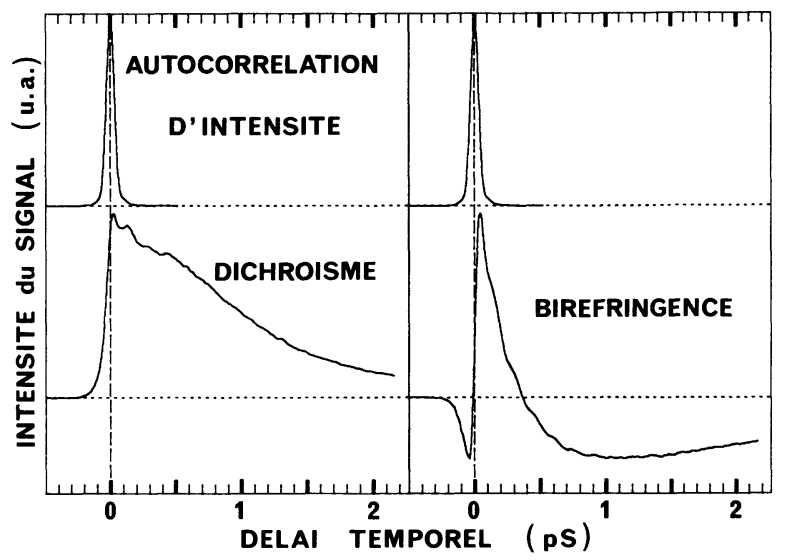

Fig. 2. - a) Mesure du dichroïsme photoinduit par excitation-sondage dans le vert de malachite en solution dans l'eau $\left(2 \times 10^{-4} \mathrm{M}\right)$. Les battements ayant une période de 150 fs déjà observés par Rosker et al. peuvent être discernés aux temps courts. En haut, la fonction d'autocorrélation $C(t)$ mesurée par somme de fréquence dans un cristal non linéaire donne la résolution temporelle de l'expérience ( $\simeq 80$ fs FWHM ). b) Mesure de la biréfringence photoinduite dans le même échantillon. Là aussi, des battements peuvent être discernés. Leur faible amplitude, comparée à la grandeur du signal plus lentement variable sur lequel elles sont superposées, empêchent une étude quantitative. La variation de l'intensité mesurée est de quelques pourcent.

[a) Photoinduced dichroism analysed in a pump-probe experiment for malachite green in a water solution $\left(2 \times 10^{-4} \mathrm{M}\right)$. Beating with a $150 \mathrm{fs}$ period previously observed by Rosker et al. can be distinguished at short times. On top, the intensity autocorrelation function $C(t)$ (Eq. (2)) measured by frequency doubling gives the experimental time resolution ( $\simeq 80$ fs FWHM, determined by the $55 \mathrm{fs}$ pulse width for a $\operatorname{sech}^{2}$ shape). b) Measurement of the induced birefringence in the same sample. Again beating can be distinguished superimposed on a slowly varying signal. Small amplitude precludes a quantitative analysis. The absorption changes measured are only a few percent.] 
d'autocorrélation d'intensité $C(\tau)$ dont la largeur à mi-hauteur ( $\simeq 80 \mathrm{fs}$ ) donne la résolution temporelle de l'expérience. Superposée à la variation complexe non exponèntielle des signaux obtenus, des battements ayant une période proche de $150 \mathrm{fs}$ sont discernables. Ayant déjà été observés en absorption saturée $[5,7]$, ils sont rapportés ici pour la première fois en effet Kerr (Fig. 2b). La difficulté d'analyser ces oscillations amorties dans ces expériences effectuées pourtant avec un très grand rapport signal sur bruit a motivé la recherche d'une technique plus performante.

\section{Technique de modulation du délai temporel et détection harmonique.}

Basée sur des principes exploités de longue date dans le domaine de la spectroscopie [8], la technique que nous avons mise au point repose sur la modulation à la fréquence $\Omega$ du délai temporel $\tau$ d'une amplitude $\Delta \tau$ comparable ou inférieure à la durée des impulsions lumineuses accompagnée de la détection synchrone de la modulation du signal lumineux à la fréquence $2 \Omega$. Si l'on fait un développement limité de $S(\tau)$, on obtient le signal :

$$
\begin{aligned}
S(\tau+\Delta \tau \cos (\Omega t))= & S(\tau)+\frac{\mathrm{d} S}{\mathrm{~d} \tau} \Delta \tau \cos \Omega t+ \\
& +\frac{\mathrm{d}^{2} S}{\mathrm{~d} \tau^{2}} \frac{\Delta \tau^{2}}{2} \cos ^{2} \Omega t+\cdots
\end{aligned}
$$

En regroupant les termes en fonction de leur fréquence d'oscillation $(0, \Omega, 2 \Omega, \ldots)$ jusqu'au terme harmonique :

$$
\begin{aligned}
& S(\tau+\Delta \tau \cos (\Omega t)) \simeq S(\tau)+\frac{\mathrm{d}^{2} S}{\mathrm{~d} \tau^{2}} \frac{\Delta \tau^{2}}{4}+0\left(\Delta \tau^{4}\right) \\
& +\cos (\Omega t)\left(\frac{\mathrm{d} S}{\mathrm{~d} \tau} \Delta \tau+\frac{\mathrm{d}^{3} S}{\mathrm{~d} \tau^{3}} \frac{\Delta \tau^{3}}{8}+0\left(\Delta \tau^{5}\right)\right)+ \\
& +\cos (2 \Omega t)\left(\frac{\mathrm{d}^{2} S}{\mathrm{~d} \tau^{2}} \frac{\Delta \tau^{2}}{4}+\frac{\mathrm{d}^{4} S}{\mathrm{~d} \tau^{4}} \frac{\Delta \tau^{4}}{48}+0\left(\Delta \tau^{6}\right)\right)
\end{aligned}
$$

où $0\left(\Delta \tau^{n}\right)$ désigne un terme d'ordre $\Delta \tau^{n}$.

La détection synchrone à la fréquence $\Omega$ donnera un signal $S_{\mathrm{F}}(\tau)$ proportionnel à la dérivée première $\mathrm{d} S(\tau) / \mathrm{d} \tau$. Déjà utilisée [9], cette observation n'a pas permis d'obtenir une amélioration notable du rapport signal sur bruit d'une expérience. La détection synchrone à la fréquence harmonique $2 \Omega$ donne accès à $S_{\mathrm{H}}(\tau)=\mathrm{d}^{2} S(\tau) / \mathrm{d} \tau^{2}$, si $\Delta \tau$ est assez faible pour que les termes d'ordre 4 et supérieurs en $\Delta \tau$ soient faibles devant le terme en $\Delta \tau^{2}$. Dans cette approximation, en prenant par commodité le signe opposé :

$$
\begin{aligned}
S_{\mathrm{H}}(\tau) & \propto \frac{-\mathrm{d}^{2}}{\mathrm{~d} \tau^{2}} \int_{-\infty}^{\infty} R\left(t^{\prime}\right) C\left(\tau-t^{\prime}\right) \mathrm{d} t^{\prime} \\
& \propto \int_{-\infty}^{\infty} R\left(t^{\prime}\right) \frac{-\mathrm{d}^{2}}{\mathrm{~d} \tau^{2}} C\left(\tau-t^{\prime}\right) \mathrm{d} t^{\prime} .
\end{aligned}
$$

Ainsi la détection de $S_{\mathrm{H}}(\tau)$ permet d'étudier la fonction réponse $R(t)$, mais avec la fonction d'appareil $C_{\mathrm{H}}(t)=-\mathrm{d}^{2} C(t) / \mathrm{d} \tau^{2}$ symétrique et dont l'intégrale est nulle. Là encore la mesure indépendante par somme de fréquences de cette fonction d'appareil est obtenue en utilisant la même technique de modulation. La sélectivité de la mesure apparaît clairement en considérant la transformée de Fourier $S_{\mathrm{H}}(\omega)$ de $S_{\mathrm{H}}(t)$ :

$$
S_{\mathrm{H}}(\omega)=\int_{-\infty}^{\infty} \exp (i \omega \tau) S_{\mathrm{H}}(\tau) \mathrm{d} \tau
$$

avec

$$
S_{\mathrm{H}}(\tau)=\int_{-\infty}^{\infty} R\left(t^{\prime}\right) C_{\mathrm{H}}\left(\tau-t^{\prime}\right) \mathrm{d} t^{\prime}
$$

on obtient directement :

$$
S_{\mathrm{H}}(\omega)=R(\omega) \times \omega^{2} C(\omega)
$$

alors que le spectre de $S(\tau)$ vaut :

$$
S(\omega)=R(\omega) \times C(\omega) .
$$

La fonction réponse $R(\omega)$ est ainsi filtrée par $C(\omega)$ lors de la mesure directe et par $\omega^{2} C(\omega)$ lors de la mesure par notre technique de modulation. La figure 3 illustre cette différence pour une fonction de

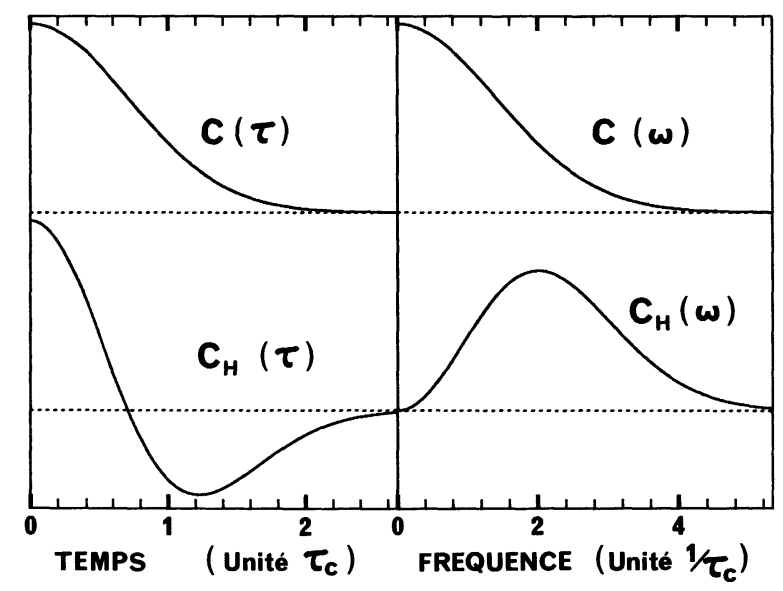

Fig. 3. - Illustration sur le modèle d'une fonction d'autocorrélation $C(\tau)=\exp \left(-\tau^{2} / \tau_{\mathrm{c}}^{2}\right)$ gaussienne, du filtrage effectué par la technique de modulation du délai temporel $\tau$ et détection synchrone du signal à la fréquence harmonique. La fonction d'appareil $C_{\mathrm{H}}(\tau)$ reste symétrique mais détermine une sélectivité en fréquence avec un maximum pour $\omega \tau_{\mathrm{c}} \simeq 2$ et une coupure totale des fréquences basses.

[Illustration on the model of a Gaussian intensity autocorrelation function $C(\tau)=\exp \left(-\tau^{2} / \tau_{\mathrm{c}}^{2}\right)$ of the filtering given by our technique of time delay $(\tau)$ modulation and synchronous detection of the signal at the harmonic frequency. The apparatus function $C_{\mathrm{H}}(\tau)=$ $-\mathrm{d}^{2} C(\tau) / \mathrm{d} \tau^{2}$ is symmetrical and has a zero integral. It determines a selectivity in frequency with a maximum for $\omega \tau_{\mathrm{c}} \simeq 2$ and a cut-off for low frequencies.] 
corrélation modèle $C(\tau)=\exp \left(-\tau^{2} / \tau_{\mathrm{c}}^{2}\right)$. Alors que la mesure directe par $C(\omega)$ permet de détecter dans $R(\omega)$ les fréquences depuis le continu jusqu'à une coupure pour $\omega \tau_{\mathrm{c}} \simeq 2$, la mesure par $C_{\mathrm{H}}(\omega)$ élimine complètement les basses fréquences et donne une sensibilité maximale pour $\omega \tau_{\mathrm{c}}=2$. Pratiquement une durée d'impulsion déterminant une largeur de $80 \mathrm{fs}$ FWHM pour $C(\tau)$ donnera lieu à un maximum de sensibilité autour d'une fréquence de $5,5 \mathrm{THz}$ (180 fs de période) et coupera les basses fréquences jusqu'à $2,3 \mathrm{THz}$ (à $1 / \mathrm{e})$.

En éliminant les signaux lents de $S(\tau)$, la présente technique élimine par là même le bruit qui les accompagne, améliorant ainsi considérablement la détectivité pour les réponses à haute fréquence. A noter que l'expérience donne directement accès au signal filtré $S_{\mathrm{H}}(\tau)$, le résultat est différent du calcul de la dérivée seconde sur le signal direct qui ne peut qu'améliorer la visualisation d'un comportement à haute fréquence [10] sans améliorer la détectivité pour ce signal.

\section{Mise en ouvre et résultats.}

Dans notre expérience, le délai temporel est modulé sinusoïdalement à l'aide d'un miroir mobile (MM sur la Fig. 1) dont l'oscillation est excitée à résonance par une cale piézoélectrique à $250 \mathrm{~Hz}$. L'amplitude de l'oscillation $\Delta t$ est ajustée à une valeur assez faible $(\Delta t \simeq 25 \mathrm{fs})$ pour que le terme en $\Delta \tau^{2}$ soit prédominant dans l'équation (4) par rapport aux termes d'ordres supérieurs (par plus d'un facteur 10). La détection synchrone du signal différence entre les deux détecteurs de mesure, à la fréquence de $500 \mathrm{~Hz}$, est aisée en raison de l'absence de signal parasite à cette fréquence.

Dans des conditions d'intensité identiques à celles ayant conduit à la figure 2 , ont été effectuées les mesures de dichroïsme induit et de biréfringence induite. La figure 4 donne les résultats de ces mesures. En haut, la fonction d'appareil $C_{\mathrm{H}}(\tau)$ est mesurée par somme de fréquences. Au centre, les courbes $S_{\mathrm{H}}(\tau)$ manifestent clairement un comportement oscillant après un pic matérialisant le recouvrement temporel des impulsions d'excitation et de sondage. Les oscillations amorties apparaissent, que ce soit pour l'absorption saturée (a) ou la biréfringence induite (b). Le rapport signal sur bruit de l'expérience est suffisant pour permettre une dilatation de l'échelle (en bas) qui fait apparaître clairement un grand nombre d'oscillations. L'exploitation de ces résultats peut se faire de manière plus simple et plus sûre que sur les signaux directs de la figure 2 . Ainsi, par exemple, il apparaît que le temps d'amortissement des oscillations d'effet Kerr est plus long que celui concernant l'absorption induite. La période d'oscillation $(\sim 150 \mathrm{fs})$ coïncidant avec le mode de vibration le plus bas de la molécule [11], l'origine du

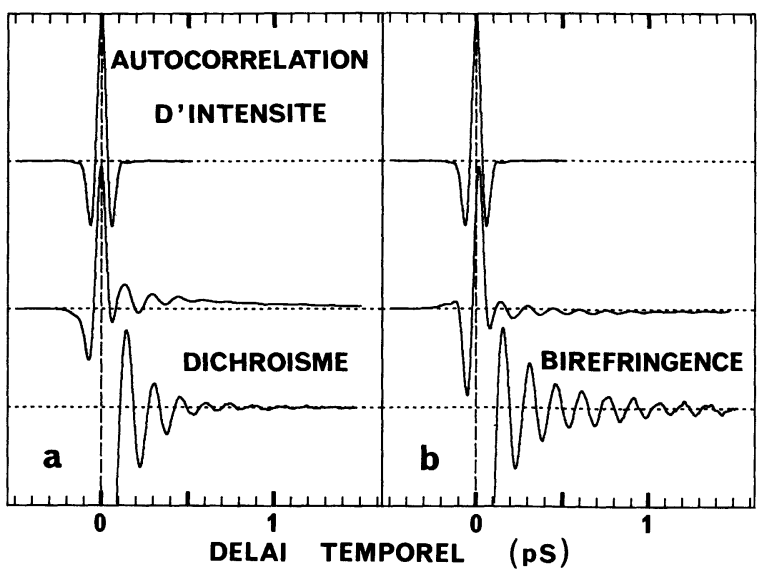

Fig. 4. - Résultats obtenus par la présente technique de modulation du délai temporel - en a, pour le dichroïsme, - en $b$, pour la biréfringence, induits dans le colorant vert de malachite en solution dans l'eau. les conditions sont identiques à celles de la figure 2 . Sous la fonction d'appareil $C_{\mathrm{H}}(\tau)$ mesurée par somme de fréquence (en haut), le signal (au milieu) montre un comportement oscillatoire après un pic. Le rapport signal sur bruit permet l'amplification de ces oscillations (en bas) et peut en permettre une analyse détaillée (le fond résiduel est filtré numériquement).

[Results obtained by the present time delay modulation technique - in a, for induced dichroism, - in b), for induced birefringence in the dye malachite green in water solution. The experimental conditions are identical to that in figure 2. Under the apparatus function $C_{\mathrm{H}}(\tau)$ (top), obtained by sum frequency generation, the signal (middle) displays an oscillatory behaviour following a peak. The signal to noise ratio allows the amplification of these oscillations (bottom) to perform a detailed analysis (the residual background is substracted numerically).]

signal est vibrationnelle. Une analyse détaillée de ces expériences de battements quantiques peut être entreprise en considérant l'effet Raman stimulé résonant en régime impulsif [12] dans lequel sont impliqués l'état électronique de base et le premier état excité. Ces résultats, ainsi que d'autres obtenus dans des conditions différentes, seront interprétés en détail dans un article séparé.

\section{Conclusion.}

Une technique simple a été mise au point pour permettre d'analyser dans une expérience d'excitation-sondage une composante à haute fréquence de la fonction réponse d'un système matériel. Cette technique nous a permis d'observer pour la première fois des battements à plusieurs térahertz pour l'effet Kerr optique dans un colorant, le vert de malachite, pour lequel Rosker, Wise et Tang avaient observé un comportement analogue en absorption saturée. Cette technique pourrait faciliter l'observation d'autres phénomènes ultrarapides superposés à un important fond lentement variable, tels qu'ils exis- 
tent pour les battements quantiques de fluorescence ou plus simplement dans le cas d'une expérience où serait présent un important fond parasite ayant par exemple comme origine la diffusion lumineuse ou un phénomène thermique comme dans le cas des réseaux transitoires induits.

\section{Bibliographie}

[1] IPPEN, E. P. and SHANK, C. V., in Ultrashort light pulses, Ed. S. L. Shapiro (Springer Verlag) 1977.

[2] Weiner, A. M. and IpPen, E. P., Chem. Phys. Lett. 114 (1985) 456.

[3] Greene, B. I. and Farrow, R. C., J. Chem. Phys. 77 (1982) 4779.

[4] Etchepare, J., Grillon, G., Thomazeau, I., Migus, A. and Antonetti, A., J. Opt. Soc. Am. B 2 (1985) 649.

[5] Rosker, M. J., Wise, F. W. and Tang, C. L., Phys. Rev. Lett. 57 (1986) 321.

[6] Chesnoy, J. and Fini, L., Opt. Lett. 11 (1986) 635.

[7] Wise, F. W., Rosker, M. J. and TANG, C. L., J. Chem. Phys. 86 (1987) 2827.
[8] Cordona, M., Modulation spectroscopy, Solid state physics (Academic Press, New-York) 1969.

[9] Taylor, A. J., Erskine, D. J. and TANG, C. L., Chem. Phys. Lett. 103 (1984) 430.

[10] Екimov, A. I., OnNShChenko, A. A., and EFros, A. L., J.E.T.P. Lett. 43 (1986) 377.

[11] Voir les commentaires de NeLson, K. A. and WILLiAms, R. L., Phys. Rev. Lett. 58 (1987) 745 ; et de

Ha, J. M. Y., MARis, H. J., Risen, W. M., TAuC, J. J., Thomsen, C. and Vardeny, Z., Phys. Rev. Lett. 57 (1986) 3302.

[12] Yan, Y. X., Gamble, E. B. and Nelson, K. A., J. Chem. Phys. 83 (1985) 5391. 